\title{
Admission control policy for adaptive multirate multiservices in cellular systems
}

\author{
Jian-Hong Wang ${ }^{*}$ and Jen-Yi Pan
}

\begin{abstract}
Forced termination of connections during handover and blocked connection initiation are annoying from the perspective of multiservice cellular system users. Previous studies have shown that an admission control policy reduces the dropping probability to a much lower level but at the cost of raising the blocking probability to a higher level. As an alternative for reducing both blocking and dropping probabilities, we make use of the Adaptive Multirate (AMR) scheme, which is a well-known real-time streaming coding technique. For an example of AMR technique, a video source is encoded into multiple independent descriptions. A cellular device, depending on its available computing and network resources, joins different descriptions to meet performance requirements. The base layer is received and the enhancement layer(s) are abandoned if the cellular capacity is insufficient for high-quality video. Mobile cellular devices currently obtain basic video quality at a lower frame rate. AMR services can substantially improve the degree of user satisfaction and guarantee the connection-level quality of service (QoS) for different multimedia. In cellular systems, the QoS requirements of different services require a connection admission control (CAC) that limits the number of connections in each access network. Therefore, we focus on the CAC and connection-level QoS of multiservice traffic using adaptive coding in mobile cellular systems. We initially analyzed our CAC policy in a multiservices cellular system by formulating the CAC policy functions, arrival rate, departure rate, blocking probability, and dropping probability of the multiservices, before we derived the connection-level QoS and verified it by simulation. The adaptive coding of multiservices traffic has a significant impact on the connection-level QoS in multiservice cellular systems. Our method decreased the blocking and dropping probabilities by adapting multirate services while users were roaming. Mobile cellular operators providing AMR services could apply our CAC to fulfill the quality requirements of the user experience and also improve the connection-level QoS.
\end{abstract}

Keywords: Connection admission control (CAC), Mobile cellular systems, Adaptive coding of multiservices traffic, Quality of service (QoS), Blocking and dropping probabilities

\section{Introduction}

Cellular systems provide wide-area network coverage. Users move within the coverage areas and they will switch connections among cellular systems according to their roaming agreements. The process of switching connections among cellular systems is known as a handoff or handover.

The evolution of wireless cellular systems over the last decade has created a demand for multimedia services with guaranteed levels of Quality of Service (QoS).

\footnotetext{
* Correspondence: wwwccucomtw@gmail.com

Department of Communications Engineering, National Chung Cheng University, Chiayi, Taiwan.
}

Connection admission control (CAC) schemes are used to selectively limit the number of admitted calls from each traffic class to maximize network utilization while satisfying the QoS constraints [1]. In a cellular system, the QoS requirements for different services (e.g., voice, real-time video) require a $\mathrm{CAC}$ to limit the number of connections in each access network [2]. The CAC policy can either accept the connection request and allocate the resources accordingly or reject the connection request. In general, higher priority is given to the requests from handover users rather new users. From the user perspective, abrupt termination of a connection is more annoying than occasional blocking of new connection attempts.

\section{实 Springer}


Standard CAC schemes include a Guard Channel (GC), Queuing Priority (QP), cutoff priority (CP) [3], the fractional guard channel (FG) schemes [4], and multiple threshold schemes. Most studies [4,5] only consider a single-service class. They assume that all arriving connections request the same amount of bandwidth. Therefore, CAC schemes have been proposed for multiservice wireless networks by Chao and Chen [6], Stratogiannis et al. [2], and Stevens-Navarro et al. [7].

Chao and Chen [6] investigated the CAC problem with mobile personal communications networks. Their study focused on the CAC used for multiple-class calls with user mobility. A generic class of coordinate-convex CAC policies was considered.

Stratogiannis et al. [2] developed a scheme gradually suppresses the admission rate of the new calls of each service class (SC), supported considering their priorities independently. The scheme was examined both for a single SC and for multiple SCs under general conditions [2].

Stevens-Navarro et al. [7] evaluated four different combinations of CAC policy by extending the $\mathrm{CP}$ and FG admission control policy with policy functions. Applying the $\mathrm{CP}$ policy to both access networks achieved the best performance given a wide range of connection request rates and various user mobility levels. The $\mathrm{CP}$ policy reserved a fixed number of channels for connection requests from handover users. Connection requests from new users were blocked if an unreserved channel was not available. $\mathrm{CP}$ admission control policy and network performance were evaluated in terms of the blocking and dropping probabilities for new and handover connections.

However, previous studies of call blocking and dropping performance with CAC have not considered adapting multiservices in cellular systems. Mobile cellular systems can now support multiple call services, such as voice and video calls. The bandwidth of these call services is usually fixed. If the bandwidth of these call services can be adapted, the CAC used by such adaptive multirate (AMR) services may reduce the call blocking and dropping probabilities, thereby increasing cellular system utilization to meet a guaranteed level of QoS. For example, G.722 can change the bandwidth of voice call services while Multiple Description Coding (MDC) can vary the bandwidth of video call services.

Various models and CAC policies have been proposed in the literature, but this article is motivated by the following specific issues.

(1) Support of multiple SCs with different bandwidth requirements.

(2) Support of adaptive bandwidth for call services.

(3) Effects of using our CAC policy in wireless access networks.
This study evaluated the CAC policy with an AMR services cellular system. We introduced the CAC policy functions that were based on the service category (e.g., voice, video, and voice call) and the type of connection request (i.e., a new request or a handover request). Initially, we developed an analytical model to evaluate the CAC in an AMR services cellular system. We evaluated the system performance when the CAC policy was in use.

The contributions of this article are as follows.

(1) We evaluated the effect of using a CAC policy, which was supported by adaptive bandwidth for call service in wireless access networks.

(2) We evaluated the performance of the cellular systems using a policy based on the arrival rates of connection requests.

The remainder of this article is organized as follows. The AMR method is introduced in Section II. The analytical model for cellular systems and CAC are described in Section III. Numerical results are presented and discussed in Section IV. Finally, our conclusions are stated in Section V.

\section{AMR service application}

Recent advances in voice and video compression techniques have led to an increasing interest in live voice and video services. In this section, we introduce rate adaptation coding techniques used for multiservice cellular systems. We consider the issues associated with adaptive voice and video mechanisms. A review of dynamic schemes used for the adaptation of voice encoders in cellular networks is also provided. The video source is encoded into multiple independent descriptions. Mobile cellular operators depend on the available cellular bandwidth to join different descriptions and meet the cellular bandwidth requirements. The introduction of an AMR codec for voice services and MDC of video service is conducted as follows.

\section{AMR voice coding}

The AMR codec for voice services was the fourth speech compression algorithm to be standardized by the European Telecommunications Standards Institute (ETSI) [8]. The third-generation WCDMA system took a novel and a flexible approach to AMR. The compression algorithm was identical for both GSM and WCDMA, and it decreased identical source encoding rates of 12.2 down to $4.75 \mathrm{~kb} / \mathrm{s}$ [9].

AMR has two traffic channel modes in a global system for mobile communication (GSM): adaptive full-rate speech (AFS) and adaptive half-rate speech (AHS). The gross bit rate of AFS is $22.8 \mathrm{~kb} / \mathrm{s}$, whereas that of AHS is $11.4 \mathrm{~kb} / \mathrm{s}$. The gross bit rate is the sum of the speech 
codec bit rate and the channel codec [8]. A mobile station equipped with AMR can request a particular mode, subject to the approval of the base station.

\section{AMR video coding}

MDC is an AMR video coding method. The MDC increases the connection efficiency when communicating video over a telephone network. MDC is a coding technique that fragments a single media stream into $\mathrm{n}$ substreams $(n \geq 2)$, which are referred to as descriptions. The packets of each description are routed over multiple paths. The MDC is a form of data partitioning used in MPEG-2 and MPEG-4. The MDC is also a layered coding mechanism that generates a base layer and $n$ enhancement layers. The base layer provides the basic video quality at a lower frame rate. Adding an enhancement layer to the base layer increases the smoothness of video quality. The enhancement layer(s) is useless for a receiver if the base layer is lost. The receiver obtains basic video quality with a lower frame rate if only the base layer is received and the enhancement layer(s) are lost. The two parts are received and combined to provide the usual high-quality video [10]. The I- and P-frames belong to the base layer, while the B-frames belong to the enhancement layer. The I-frame (or intra-coded frame) is encoded independently of other frames and it is decoded in isolation. The P-frame (or predictive frame) is encoded based on prediction from the preceding I- frame or a Pframe in the video sequence. The B-frame (or bidirectionally predictive-coded frame) is encoded based on prediction from preceding and succeeding I- or P-frames . In this study, a single media stream was cut into two substreams referred to as descriptions.

G.722.2 (AMR-wideband) can adapt to the bandwidth of voice call services while MDC can adapt to the bandwidth of video call services. If the bandwidth of these call services is adaptable, the CAC for such adaptive rate services can reduce the call blocking and dropping probability, and it can also increase the cellular system utilization to meet a guaranteed QoS level.

\section{System model and admission control method}

In this section, we present a model for adaptive rate multiservice cellular systems. We describe the CAC policy and we also analyze the blocking and dropping probabilities of connection requests. Figure 1 shows the multirate service cellular systems used in this study while Table 1 details some of the important symbols used in this article.

Handover can occur because of the movement of users among different cells, as shown in Figure 1. We considered a general model of multiservice calls with mobility when studying CAC policy, because the majority of the literature is concerned with multiple-class calls in fixed networks (i.e., without mobility) [11] or calls of only a single class [12].

Assume that there are a total of four cells in a cellular system and two call services. One of the services is a "single mode service" while the other is a "dual mode service". The bandwidth occupied by the "single mode service" is fixed and it cannot be adapted. The "dual mode service" has two service modes, where one is an adaptive-rate service that has not been adapted, i.e., the "dual mode full-rate service", whereas the other service mode is an adaptive-rate service that has been adapted, i.e., the "dual mode reduced-rate service".

The six conditions that used in this study, as shown in Figure 1, are as follows.

(1) "Condition 1" indicates $\sum_{s^{\prime} \in S} m_{3_{s^{\prime}}} b_{s^{\prime}}+b_{1} \leq \psi$. We denote the occupied bandwidth of cell 3 by $\sum_{s^{\prime} \in S} m_{s_{s^{\prime}}} b_{s^{\prime}}$, the connection request bandwidth of "single mode service" by $b_{1}$, and the threshold of the new connection request by $\psi$. While "Condition 1 " is satisfied, a new "single mode service" connection request is permitted from point $K$ to $L$ in cell 3 .

(2) “Condition 2" indicates $\sum_{s^{\prime} \in S} m_{4_{s^{\prime}}} b_{s^{\prime}}+b_{1} \leq C_{4}$. We denote the occupied bandwidth of cell 4 by $\sum_{s^{\prime} \in S} m_{4^{\prime} s^{\prime}} b_{s^{\prime}}$ and the total bandwidth of cell 4 by $C_{4}$. While "Condition 2" is satisfied, a "single mode service handover connection request is permitted" from point $L$ to $M$ in cell 4 . This increases the blocking probability and reduces the dropping probability.

(3) "Condition 3" indicates $\sum_{s^{\prime} \in S} m_{i_{s}} b_{s^{\prime}}+b_{2} \leq \psi$. We assume $i=2$ and we denote the occupied bandwidth of cell 2 by $\sum_{s^{\prime} \in S} m_{s_{s}^{\prime}} b_{s^{\prime}}$ and the connection request bandwidth of "dual mode full-rate service" by $b_{2}$. While "Condition 3" is satisfied, a new "dual mode full-rate service" connection request is permitted from point $J$ to $D$ in cell 2 . Similarly, both the new "dual mode full-rate service" connection requests from point $A$ to $B$ in cell 1 and from point $A$ to $E$ in cell 4 follow "Condition 3".

(4) "Condition 4" indicates $\sum_{s^{\prime} \in S} m_{i^{\prime}} b_{s^{\prime}}+b_{2} \leq \sigma$. We assume $i=2$ and we denote the threshold of the handover connection request by $\sigma$. While "Condition 4 " is satisfied, a "dual mode full-rate service" handover connection request is permitted from point $B$ to $D$ in cell 2 . Similarly, the "dual mode fullrate service" handover connection request from point $E$ to $F$ in cell 1 follows "Condition 4".

(5) "Condition 5" indicates $\sigma<\sum_{s^{\prime} \in S} m_{s_{s^{\prime}}} b_{s^{\prime}}+b_{2} \leq C_{2}$. We denote the total bandwidth of cell 2 by $C_{2}$. While the "Condition 5" is satisfied, the "dual mode 


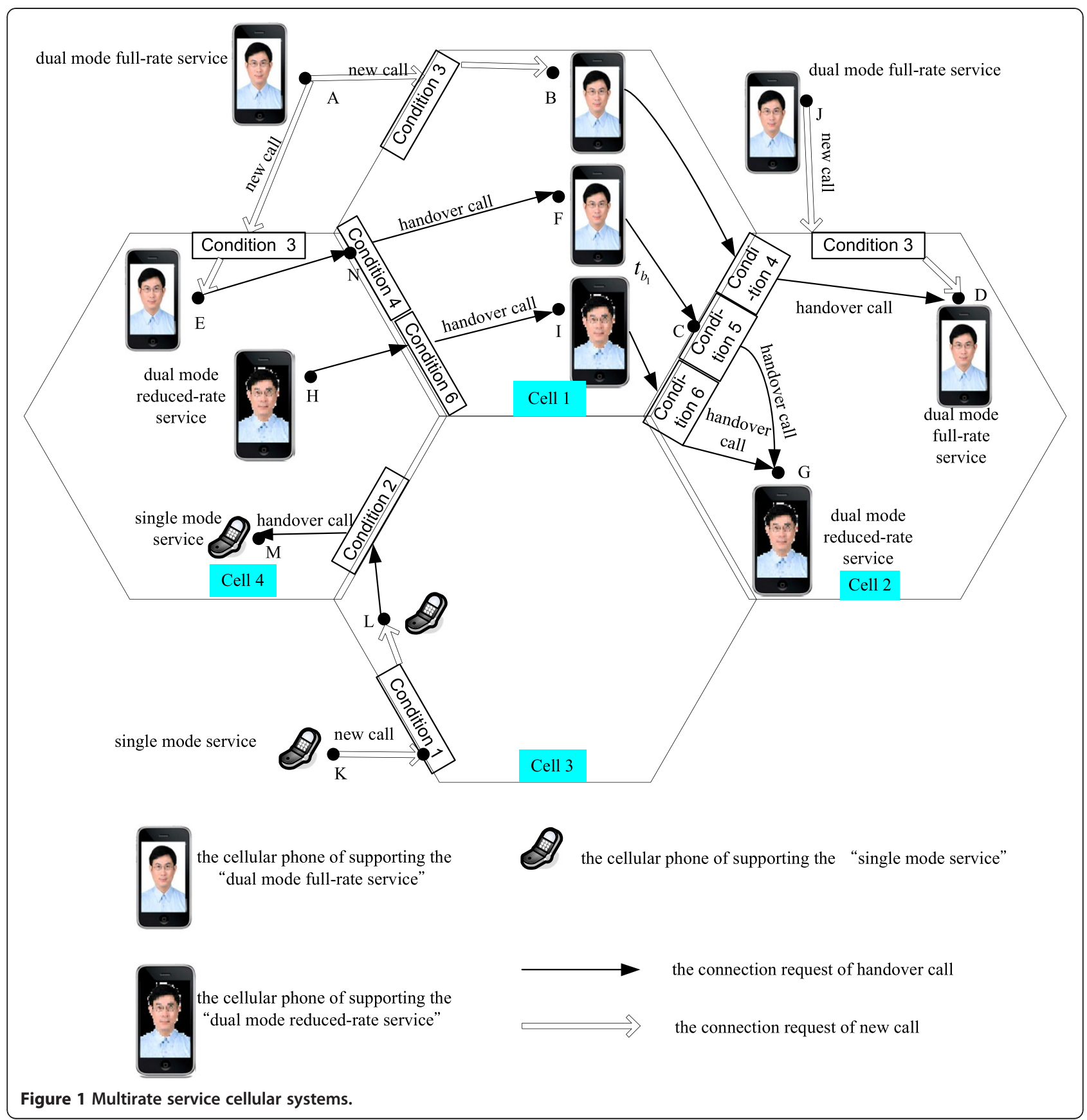

full-rate service" in point $F$ of cell 1 changes into the "dual mode reduced-rate service" in point $G$ of cell 2 , and the handover connection request from point $F$ to $G$ in cell 2 is permitted. The location of the service type change is point $C$.

(6) "Condition 6" indicates $\sum_{s^{\prime} \in S} m_{i^{\prime}} b_{s^{\prime}}+b_{2^{*}} \leq C_{i}$. We assume $i=2$ and the connection request bandwidth of "dual mode reduced-rate service" by $b_{2 *}$. While "Condition 6" is satisfied, a "dual mode reduced-rate service" handover connection request is permitted from point $I$ to $G$ in cell 2 . Similarly, the "dual mode reduced-rate service" handover connection request from point $H$ to $I$ in cell 1 follows "Condition 6".

Those conditions comprise the CAC policy function and they aim to simultaneously reduce the blocking and dropping probabilities of each service in multi-service cellular systems.

\section{Traffic and mobility models}

We define the interboundary time to model mobility, in a similar way to [13], which is the time interval between any two consecutive access network boundary crossings 
Table 1 Symbols used in this article and their definitions

\begin{tabular}{|c|c|}
\hline Symbol & Explanation \\
\hline $\bar{M}$ & The set of all cells in a wireless cellular system \\
\hline$A_{i}$ & The set of cells that are adjacent to cell $i$ \\
\hline$S$ & $\begin{array}{l}\text { The set of multimedia services that are available to } \\
\text { mobile users }\end{array}$ \\
\hline$s$ & The multimedia services that are available to mobile users \\
\hline$S_{\text {single-mode }}$ & $\begin{array}{l}\text { The set of "single mode services" that are not } \\
\text { adaptive-rate services }\end{array}$ \\
\hline$S_{\text {full-rate }}$ & $\begin{array}{l}\text { The set of "dual mode full-rate services" that are } \\
\text { adaptive-rate services, but which have not been adapted }\end{array}$ \\
\hline$S_{\text {reduced-rate }}$ & $\begin{array}{l}\text { The set of "dual mode reduced-rate services" that } \\
\text { are adaptive-rate services, and which have been adapted }\end{array}$ \\
\hline $\operatorname{MDC}(s)$ & $\begin{array}{l}\text { A single media stream of the "dual mode full-rate } \\
\text { service" that can be cut into two substreams referred } \\
\text { to as descriptions (i.e., "dual mode reduced-rate service") }\end{array}$ \\
\hline$b_{s}$ & The basic bandwidth unit (BBU) of each service, \\
\hline$t_{5}$ & $\begin{array}{l}\text { The connection time, which is the duration of the } \\
\text { connection using service type } s \text { and an exponentially } \\
\text { distributed random variable with mean } 1 / v_{s}\end{array}$ \\
\hline$t_{s}^{R}$ & $\begin{array}{l}\text { The residual (i.e., remaining) connection time, which is } \\
\text { exponentially distributed with mean } 1 / v_{s}\end{array}$ \\
\hline $1 / v_{s}$ & The mean of $t_{s}$ \\
\hline$t_{b_{i}}$ & $\begin{array}{l}\text { The interboundary time, which starts at the moment } \\
\text { cell } i \text { is entered and that is an exponentially } \\
\text { distributed random variable with means } 1 / n_{i}\end{array}$ \\
\hline $1 / \eta_{i}$ & The mean of $t_{b_{i}}$ \\
\hline$q_{i_{s} j_{s}}$ & $\begin{array}{l}\text { The probability of attempting a handover of service } \\
\text { type } s \text { from cell } i \text { to neighboring cell } j\end{array}$ \\
\hline$m_{i}$ & The occupancy vector for overall services in cell $i$ \\
\hline$m_{i_{s}}$ & $\begin{array}{l}\text { The number of connections using multimedia service } \\
\text { type } s^{\prime} \text { in cell } i\end{array}$ \\
\hline$\theta_{i}$ & The set of all feasible $m_{i}$ vectors \\
\hline$\lambda_{i_{s}}$ & $\begin{array}{l}\text { The arrival rate of the new connection request for } \\
\text { service type } s \text { in cell } i\end{array}$ \\
\hline$P_{i}\left(m_{i}\right)$ & $\begin{array}{l}\text { The probability of being in state } m_{i} \text { in the }|S| \text {-dimensional } \\
\text { birth-death process corresponding to cell } i\end{array}$ \\
\hline$C_{i}$ & The capacity for cell $i$ \\
\hline$\psi$ & The threshold of a new connection request \\
\hline$\sigma$ & The threshold of a handover request \\
\hline$\beta_{n_{i s}}\left(m_{i}\right)$ & $\begin{array}{l}\text { The policy of not accepting a new user's connection } \\
\text { request for service type } s \text { in cell } i\end{array}$ \\
\hline$\beta_{h h_{i s t}}\left(m_{i}\right)$ & $\begin{array}{l}\text { The policy of not accepting a connection request } \\
\text { from a handover user for service type } t \text {, which is } \\
\text { offered to cell } i \text { from service type } s \text { in its adjacent cell }\end{array}$ \\
\hline$B_{n_{i s}}$ & $\begin{array}{l}\text { The probability of blocking connection requests for } \\
\text { service type } s \text { in cell } i \text { of new users }\end{array}$ \\
\hline$B_{h h_{i s t}}$ & $\begin{array}{l}\text { The probability of dropping connection requests for } \\
\text { service type } t \text { of handover users, which is offered } \\
\text { to cell } i \text { from service type } s \text { in its adjacent cell }\end{array}$ \\
\hline$h_{j_{s} i_{s}}$ & $\begin{array}{l}\text { The handover rate of service type } s, \text { which is offered } \\
\text { to cell } i \text { from its adjacent cell } j\end{array}$ \\
\hline$h_{j_{s} i_{t}}$ & $\begin{array}{l}\text { The handover rate of service type } t \text {, which is offered } \\
\text { to cell } i \text { from service type } s \text { in its adjacent cell } j\end{array}$ \\
\hline
\end{tabular}

Table 1 Symbols used in this article and their definitions (Continued)

\begin{tabular}{ll}
\hline $\mathfrak{I}$ & The new call arrival rates after processing the \\
& CAC policy function \\
$\mathfrak{R}$ & The arrival rates of handover calls that are not \\
& adaptive-rate calls after processing the CAC policy function \\
$\partial$ & The arrival rates of handover calls that are \\
& adaptive-rate call after processing the CAC policy function
\end{tabular}

by a mobile user. The interboundary time depends on the size of the cell and the mobility patterns of the users. If an interboundary time starts at the moment cell $i$ is entered, then we denote it by $t_{b_{1}}$. We assume that $t_{b_{1}}$ is an exponentially distributed random variable with mean $1 / \eta_{i}$. Figure 1 shows $t_{b_{1}}$, which is the time between the boundary crossing points $N$ and $C$. The channel holding time in cell $i$ is defined as the time that a connected mobile user continues to use $b_{s}$ BBUs (basic bandwidth units) of resources in multi-service cellular systems.

For service type $s$, the channel holding time in cell $i$ is $\min \left(t_{s}^{R}, t_{b_{i}}\right)$. The $t_{s}^{R}$ and $t_{b_{i}}$ have exponential distributions for all $s \in S, S=S_{\text {single-mode }} \cup S_{\text {full-rate }} \cup S_{\text {reduced-rate }}, i \in$ $M$, and the holding times are also exponentially distributed with parameters $\mu_{i_{s}}=v_{s}+\eta_{i}$. A mobile user holding a connection of service type $s$ in cell $i$ may terminate this connection at the end of their holding time and leave the cellular system with probability $q_{i_{s t}}=$ $\left(1 / \eta_{i}\right) /\left(1 / v_{s}+1 / \eta_{i}\right)=v_{s} /\left(v_{s}+\eta_{i}\right)$. The user may also move within the system and continue in an adjacent cell with probability $1-q_{i_{s t}}$.

$$
1-q_{i_{s t}}=\frac{\eta_{i}}{v_{s}+\eta_{i}}=\sum_{j \in A_{i}} q_{i_{s} j_{t}}
$$

\section{Policy functions for CAC}

The capacity of the $C_{i}$ BBUs constraint requires that

$$
\begin{aligned}
\sum_{s \in S} m_{i_{s}} b_{s} \leq C_{i}, \forall i & \in M, m_{i_{s}} \geq 0, m_{i} \\
& =\left(m_{i_{1}}, m_{i_{2}}, m_{i_{3}}, \ldots, m_{i_{s}}\right)
\end{aligned}
$$

The number of connections of service type $s$ in cell $i$ is $C_{i} / b_{s}$ at any time. For each cell $i \in\{1,2,3,4\}$, the CAC policies for connection requests from new and handover users for service type $s \in S$ can be modeled using the policy functions $\beta_{n_{i s}}\left(m_{i}\right)$ and $\beta_{h h_{i s t}}\left(m_{i}\right)$, respectively. In general, higher priority is given to requests from handover users rather than new users, because a connection that is abruptly terminated is more annoying than an occasionally blocked new connection attempt from the user perspective. Therefore, handover requests have a higher priority than new requests, so it is necessary that $\beta_{n_{i s}}\left(m_{i}\right) \geq \beta_{h h_{i s t}}\left(m_{i}\right)$ for all $i \in M$ and $s \in S$. 
This section introduces the policy function concept and derives the corresponding functions for CAC policy. Stevens-Navarro et al. [7] showed that, under a wide range of connection request rates and various user mobility levels, the application of a CP policy in both access networks provided the best performance with both design objectives. Therefore, this study uses CP policy functions for CAC. The CP policy reserves a fixed number of available channels (i.e., BBUs) for handover requests. Next, using the notation of policy functions, a connection request to cell $i$ for service type $s$ is rejected by the CP policy for new users:

$$
\beta_{n_{i_{s}}}\left(m_{i}\right)=\left\{\begin{array}{c}
0, \text { if } s \in S_{\text {single-mode }}, \sum_{s^{\prime} \in S} m_{i_{s^{\prime}}} b_{s^{\prime}}+b_{s} \leq \psi \\
0, \quad \text { if } s \in S_{\text {full-rate }}, \sum_{s^{\prime} \in S} m_{i_{s^{\prime}}} b_{s^{\prime}}+b_{s} \leq \psi \\
1, \quad \text { otherwise }
\end{array}\right.
$$

of blocking connection requests for service type $s$ in cell $i$ of new users is as follows

$$
B_{n_{i s}}=\sum_{m_{i} \in \theta_{i}} P_{i}\left(m_{i}\right) \beta_{n_{i_{s}}}\left(m_{i}\right)
$$

while the probability of dropping connection requests for service type $t$ of handover users, which are offered to cell $i$ from service type $s$ in its adjacent cell is

$$
B_{h h_{i s t}}=\sum_{m_{i} \in \theta_{i}} P_{i}\left(m_{i}\right) \beta_{h h_{i s t}}\left(m_{i}\right)
$$

Let $\phi_{i_{s t}}\left(m_{i}\right)$ denote the birth rate of service type $t$, which is offered to cell $i$ from service type $s$ before entering the cell. We have

$$
\phi_{i_{s t}}\left(m_{i}\right)=\mathfrak{I}+\mathfrak{R}+\partial
$$

and the CP policy for handover users:

$$
\beta_{h h_{i s t}}\left(m_{i}\right)=\left\{\begin{array}{c}
0, \text { if } s \in S_{\text {single-mode }}, t=s, \sum_{s^{\prime} \in S} m_{i_{s^{\prime}}} b_{s^{\prime}}+b_{s} \leq C_{i} \\
0, \text { if } s \in S_{\text {reduced-rate }}, t=s, \sum_{s^{\prime} \in S} m_{i_{s^{\prime}}} b_{s^{\prime}}+b_{s} \leq C_{i} \\
0, \text { if } s \in S_{f u l l-\text { rate }}, t=s, \sum_{s^{\prime} \in S} m_{i_{s^{\prime}}} b_{s^{\prime}}+b_{s} \leq \sigma \\
0, \text { if } s \in S_{\text {full-rate }}, t=M D C(s), t \in S_{\text {reduced-rate }}, \sigma \leq \sum_{s^{\prime} \in S} m_{i_{s^{\prime}}} b_{s^{\prime}}+b_{s} \leq C_{i} \\
1, \text { otherwise }
\end{array}\right.
$$

where $\sum_{s^{\prime} \in S} m_{i_{s}} b_{s^{\prime}}$ denotes the current occupancy, the integer parameter $\psi$ in (3) is used to give priority to new requests whereas the parameter $\sigma$ in (4) is used to give priority to handover request.

\section{Birth-death processes}

An occupancy vector $m_{i}$ is feasible if $m_{i^{\prime}} \geq 0$ for all $s \in S$, and the constraint in (2) is satisfied. We denote the set of all feasible $m_{i}$ vectors by $\theta_{i}$. The occupancy of cell $i$ evolves according to a multidimensional birth-death process [14]. A birth event occurs when a connection request to cell $i$ is accepted from a handover or a new user. A death event occurs when a user either terminates its connection or leaves cell $i$. Next, let $P_{i}\left(m_{i}\right)$ denote the probability of being in state $m_{i}$ in the $|S|$-dimensional birth-death process corresponding to cell $i$. The probability where

$$
\begin{gathered}
\mathfrak{I}=\left\{\begin{array}{c}
\lambda_{i_{s}}\left(1-\beta_{n_{i s}}\left(m_{i}\right)\right), \quad \text { if } s \in S_{\text {single-mode }} \\
\lambda_{i_{s}}\left(1-\beta_{n_{i s}}\left(m_{i}\right)\right), \quad \text { if } s \in S_{\text {full-rate }} \\
0, \text { otherwise }
\end{array}\right. \\
\mathfrak{R}=\left\{\begin{array}{c}
\sum_{j \in A_{i}} h_{j_{s} i_{t}}\left(1-\beta_{h h_{i_{s t}}}\left(m_{i}\right)\right), \text { if } s \in S_{\text {single-mode }}, t=s \\
\sum_{j \in A_{i}} h_{j_{s} i_{t}}\left(1-\beta_{h h_{i_{s t}}}\left(m_{i}\right)\right), \text { if } s \in S_{\text {full-rate }}, t=s \\
0, \text { otherwise }
\end{array}\right.
\end{gathered}
$$

where $h_{j_{s} i_{t}}$ denotes the handover rate of service type $t$, which is offered to cell $i$ from service type $s$ in its adjacent cell $j$.

Let $\phi_{i_{s}}$ denote the death rate of service type $s$ in the birth-death process corresponding to cell $i$. Recall that a

$$
\partial=\left\{\begin{array}{c}
\sum_{j \in A_{i}} h_{j_{s} i_{t}}\left(1-\beta_{h h_{i t t}}\left(m_{i}\right)\right)+\sum_{j \in A_{i}} h_{j_{s} i_{s}}\left(1-\beta_{h h_{i_{s t}}}\left(m_{i}\right)\right), \quad \text { if } s \in S_{\text {full-rate }}, t=M D C(s), t \in S_{\text {reduced-rate }} \\
\text { 0, otherwise }
\end{array}\right.
$$




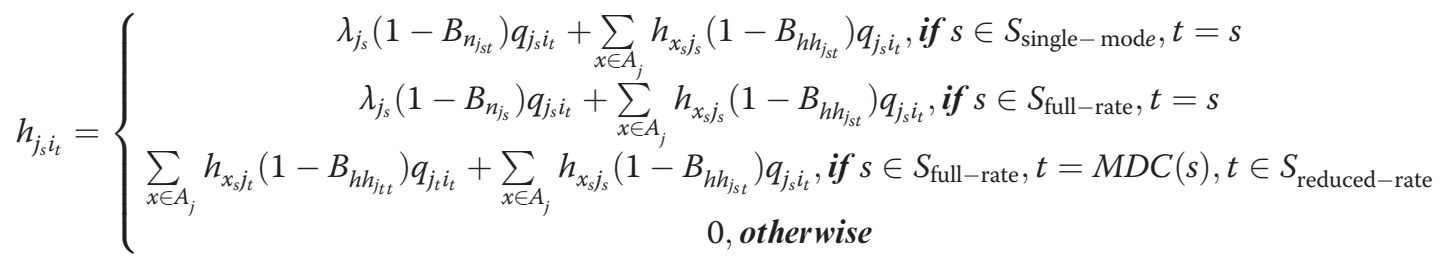

death event occurs when a user either terminates its connection or leaves cell $i$.

$$
\phi_{i_{s}}=m_{i_{s}} \mu_{i_{s}}
$$

Given the policy functions $\beta_{n_{i_{s}}}\left(m_{i}\right), \beta_{h h_{i_{s t}}}\left(m_{i}\right)$, and the network parameters $\lambda_{i_{s}}, v_{s}, \eta_{i}, \mu_{i_{s}}, q_{i_{i t_{t}}}, C_{i}$, and $b_{s}$ for all cell $i \in M$ and $s \in S$, we can solve the global balance equations of the birth-death processes and obtain the corresponding blocking probability $B_{n_{i s}}$ and dropping probability $B_{h h_{i s t}}$. To compute the birth rates in (7), we need to solve the handover rate Equations (11). To compute the blocking and dropping probabilities for connection requests from new and handover users of service $s$, the following iterative fixed-point algorithm [15] is used.

First, we set $B_{n_{i_{s}}}=0, B_{h h_{i s t}}=0$ for all cells $i \in M$ and $s \in S$. Second, if $\left\|B_{n_{i s}}\right\|+\left\|B_{h h_{i s t}}\right\|>\epsilon$ is true, then we solve the system of handover rate equations given by (11) and compute the birth rates $\phi_{i_{s t}}\left(m_{i}\right)$, the blocking probability $\overline{B_{n_{i s}}}$, and the dropping probability $\overline{B_{h h_{i s t}}}$. Third, we update $B_{n_{i s}}=\overline{B_{n_{i s}}}, B_{h h_{i s t}}=\overline{B_{h h_{i s t}}}$. Next, we backtrack the second step until the results satisfy the condition $\left\|B_{n_{i s}}\right\|+\left\|B_{h h_{i s t}}\right\|<\epsilon$. The function $\|B\|$ is defined as $\sum_{j}\left|\overline{B_{j}}-B_{j}\right| . \epsilon\left(\epsilon=10^{-5}\right)$ is a deviation value that is accepted in this study.

\section{Numerical results and discussions}

We evaluate the performance of a wireless cellular system with four cells, as shown in Figure 1. Figure 1 shows as example, where we have cell $i \in\{1,2,3,4\}, M=$ $\{1,2,3,4\}, A_{1}=\{2,3,4\}$. Each service $s \in S$ requires $b_{s}$, a BBU to guarantee its QoS requirements in the cellular system. In each cell, the network capacity is $2 \mathrm{Mb} / \mathrm{s}$, and the $\mathrm{BBU}$ is set to $32 \mathrm{~kb} / \mathrm{s}$ based on the 3GPP-supported multimedia bearer services [16]. This implies that the capacity of each cell is $C_{i}=62$ BBUs.

We assume that two multimedia services are offered with service types that have "single mode service", "dual mode full-rate service", and "dual mode reduced-rate service" (i.e. , $S=\left\{1,2,2^{*}\right\}$ ). The first service, i.e., $s=1$, is a voice connection requiring $32 \mathrm{~kb} / \mathrm{s}$. Type 1 service indicates a "single mode service". The second service, i.e., $s=2$, is a video telephone $(128 \mathrm{~kb} / \mathrm{s})$ with voice connections requiring $160 \mathrm{~kb} / \mathrm{s}$. Type 2 service indicates a "dual mode full-rate service". The adaptive coding of a type 2 service $(s=2 *)$ is a half-quality video telephone $(64 \mathrm{~kb} / \mathrm{s})$ with voice connections requiring $96 \mathrm{~kb} / \mathrm{s}[17,18]$. Type $2^{*}$ service indicates a "dual mode reduced-rate service". The values are set according to the multimedia codec for 3GPP [19]. We assume that $b_{1}$ is the bandwidth of a type 1 service, $b_{2}$ is the bandwidth of a type 2 service, and $b_{2^{*}}$ is the bandwidth of a type $2^{*}$ service.

Thus, the QoS provisioning of these cells requires that $b_{1}=1 \mathrm{BBU}, b_{2}=5 \mathrm{BBUs}$, and $b_{2^{*}}=3 \mathrm{BBUs}$. The connection duration has the means $1 / v_{1}=1 / v_{2}=8 \mathrm{~min}$. The interboundary time in each cell has the mean $1 / \eta_{i}=2$ min, for users in the wireless cellular system.

\section{Effect of increasing the threshold of adaptive coding}

Figure 2 shows the blocking probabilities of new connection requests to the two services and the dropping probabilities of handover connection requests for types 1,2 , and $2^{*}$ services in cell 1 versus the adaptive coding threshold.

$\sigma$ ranges from 0 to 62 , while the arrival rates of new connection requests from services type 1 and 2 remain constant at 0.5 connection requests per minute and $\psi=$ 50. $\mathrm{Pb} 1$ and $\mathrm{Pb} 2$ are the blocking probabilities of new

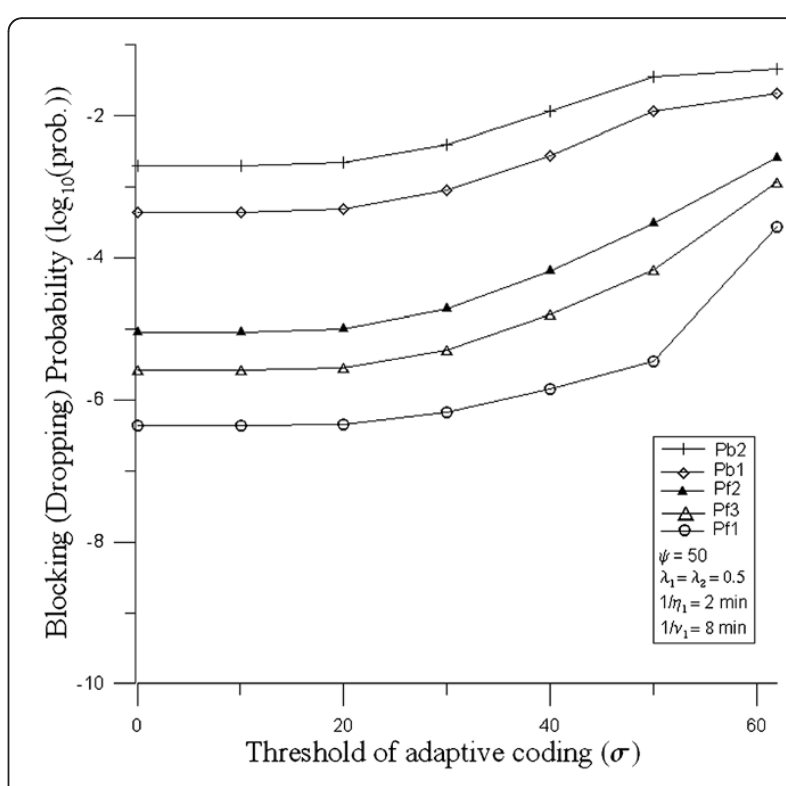

Figure 2 Blocking or dropping probability of cell 1 versus the adaptive coding threshold. 
connection requests of types 1 and 2 services, respectively. Pf1, Pf2, and Pf3 are the forced termination probabilities of handover connection requests of types 1,2 , and $2 *$ services, respectively. The blocking probabilities of new connection requests and the dropping probabilities of handover connection requests of type 2 services are the highest because the connections require five times the amount of BBUs as type 1 services. In contrast, the dropping probabilities of handover connection requests of a type $2 *$ service are higher than a type 1 service. This is because the connection requirement of a type $2^{*}$ service requires three times as many BBUs as type 1 services.

In our study, we can see that in cell 1, the new call dropping probability of a type 1 service reduces from $10^{-1.691}$ to $10^{-3.366}$ while a type 2 service reduces from $10^{-1.342}$ to $10^{-2.702}$ due to $\sigma$. Furthermore, the handover call dropping probability for a type 1 service reduces from $10^{-3.557}$ to $10^{-6.366}$, type 2 service reduces from $10^{-2.589}$ to $10^{-5.054}$, and type $2^{*}$ service reduces from $10^{-2.939}$ to $10^{-5.584}$ due to $\sigma$.

Previous studies [6] have demonstrated the effect of the level of guard capacity on blocking and forced termination probabilities. The blocking probabilities for new connection requests by two services increase whereas the dropping probabilities of handover connection requests by two services decrease when the guard capacity $\mathrm{Cg}$ changes from 0 to 48 . Previous studies [6] have reduced the forced termination probability to a very low value by allowing the blocking probability to reach a higher level.

In our study, we reduced the blocking probabilities and the forced termination probabilities to lower levels but with a loss of the QoS for video telephones with a type 2 service. The curves of two services exhibited the same trend. For example, the connection-level QoS requires that the blocking probability should be under $2 \%$ while the forced termination probability should be under $10^{-4}$. If $\sigma=62$, the blocking probabilities and the forced termination probabilities cannot satisfy the QoS requirements for both classes. However, if we retain $\sigma=40$ exclusively for handover calls, the connection-level QoS can be guaranteed for calls of both classes. The level of $\sigma$ should be determined by the connection-level QoS requirement in multi-service systems.

Figure 3 shows the adaptive coding probability for handover connection requests of with a type 2 service versus the adaptive coding threshold $(\sigma)$ of cell 1 ranging from $\psi=30$ to 50 . The adaptive coding probability in all three cases is approximately the same, whereas $\sigma<10$. It tends to one if $\sigma$ approaches to zero, as shown in Figure 3.

The permitted amount of new calls increases when $\sigma$ is fixed and $\psi$ increases. The remaining capacity of cell 1

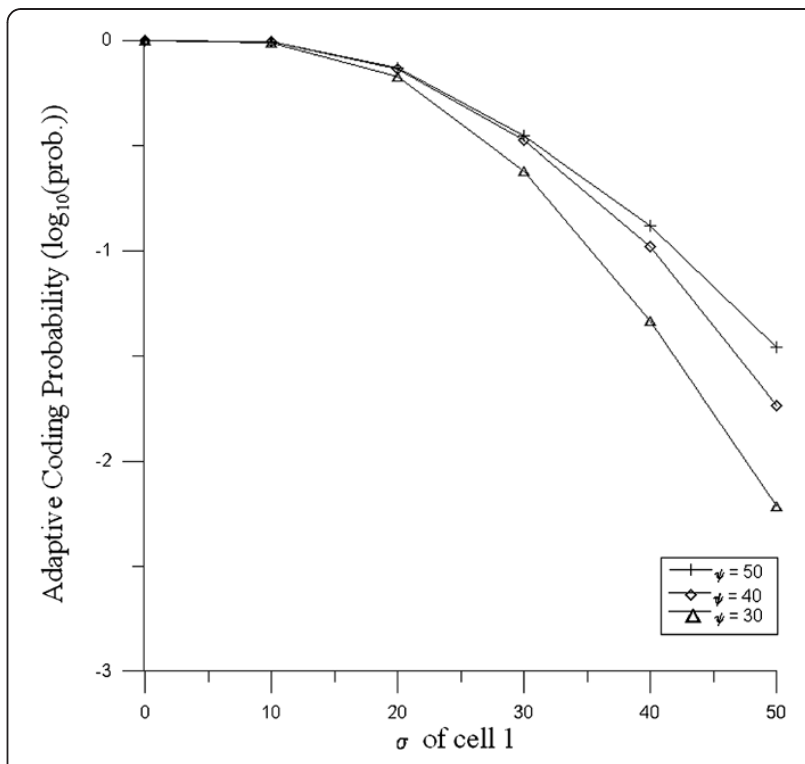

Figure 3 The adaptive coding probability versus the adaptive coding threshold $(\sigma)$ of cell 1 .

also declines. The adaptive coding probability of handover connection requests with a type 2 service gradually increases while the remaining capacity of cell 1 gradually decreases.

Figure 4 shows the average peak signal-to-noise ratio (PSNR) value when varying the adaptation coding threshold. The PSNR is used as a measure of the quality of reconstruction of lossy compression, such as video compression. The signal is the original image while the noise is the error introduced by compression. PSNR is

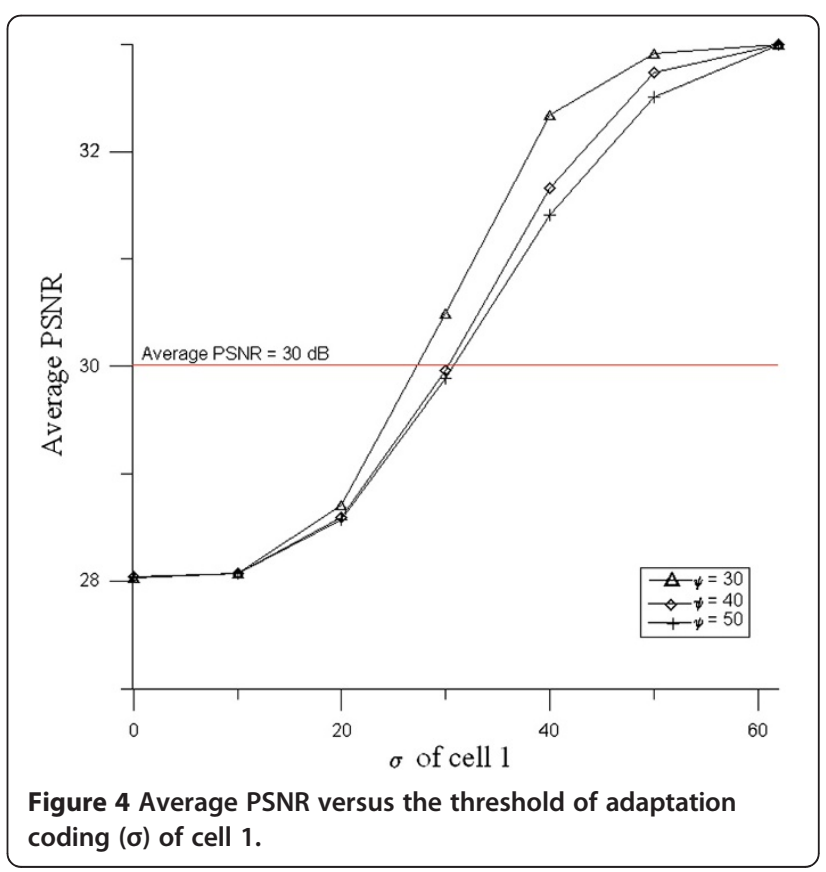


used when comparing compression codes as an approximation of the human perception of reconstruction quality, so in some cases one reconstruction may appear to be closer to the original than another, even though it has a lower PSNR. Typical values for the PSNR with lossy images and video compression are from 30 to $50 \mathrm{~dB}$. Acceptable values for wireless transmission quality loss are considered to be about 20 to $25 \mathrm{~dB}$ [20]. Therefore, the average PSNR value in Figure 4 must be above $30 \mathrm{~dB}$ to produce acceptable quality values for wireless transmission video (video with a type 2 service).

The PSNR with a type 2 service is set to $33 \mathrm{~dB}$ while it is set to $28 \mathrm{~dB}$ with a type $2^{*}$ service [21]. We assume that the number of the type 2 services in cell 1 is "n2" while the number of the type $2^{*}$ services in cell 1 are "n3". Let $\omega$ denote the average PSNR of both the type 2 and $2^{*}$ services in cell 1 . We have

$$
\omega=\left(\frac{\mathrm{n} 2}{(\mathrm{n} 2+\mathrm{n} 3)}\right) * 33 \mathrm{~dB}+\left(\frac{\mathrm{n} 3}{(\mathrm{n} 2+\mathrm{n} 3)}\right) * 28 \mathrm{~dB}
$$

The handover connection requests for type 2 services are easily changed into those of type $2^{*}$ service if the adaptation coding threshold is decreased. At this point, the average PSNR is lower while the average video quality of a type 2 service is poorer. From the other perspective, the adaptive coding probability of handover connection requests for type 2 services becomes gradually higher when $\sigma$ is fixed and $\psi$ is increased, as shown in Figure 3. The average PSNR is lower, as shown in Figure 4, while the average video quality of a type 2 service becomes very poor.

Type 2 service does not change to a type $2^{*}$ service if $\sigma=62$. At this point, the average PSNR value is $33 \mathrm{~dB}$. All handover connection requests for a type 2 service are changed into type $2^{*}$ services if $\sigma=0$. At this point, the average PSNR is $28 \mathrm{~dB}$.

\section{Varying the call to mobility ratio}

Figure 5 shows the blocking probabilities of new connection requests for types 1 and 2 services, and the dropping probabilities of handover connection requests for all services in cell 1 when the "call to mobility ratio" (CMR) of types 1 and 2 services is increased.

The CMR $\left(\boldsymbol{C M R}=\lambda_{s} / \eta_{i}\right)$ of a user is the average number of calls to a user per unit time divided by the average number of times the user changes registration areas per unit time [22-24]. We set $s \in\{1,2\}$ and $i=1$. The arrival rate of new connection requests for type $1\left(\lambda_{1}\right)$ and type $2\left(\lambda_{2}\right)$ services are set from 0 to 1 . Next, the interboundary time of each cell has a mean of $1 / \eta_{1}=2 \mathrm{~min}$ and a connection duration with means $1 / v_{1}=1 / v_{2}=8$ min. The CMR will increase and both $\lambda_{1}$ and $\lambda_{2}$ increase while the inter-boundary time in each cell is fixed. Next,

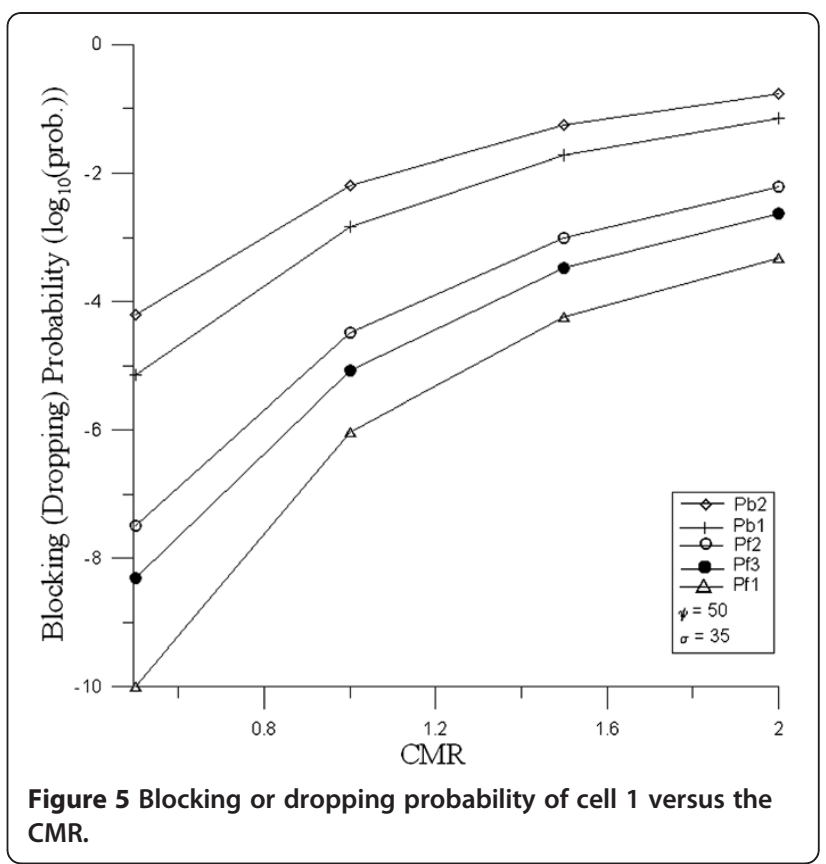

the blocking probabilities of new connection requests and the dropping probabilities of handover connection requests for the two services are increased because the occupancy of cell 1 is increased, as shown in Figure 5.

Figure 6 shows the effect of the CMR on the adaptive coding probability. $\lambda_{1}$ and $\lambda_{2}$ are set from 0 to 1 new connection requests per minute. Next, we set $1 / \eta_{1}=$ 2 min, $\psi=50$, and $\sigma$ from 35 to $62 . \lambda_{1}, \lambda_{2}$, and the occupancy of cell 1 are very low, while $\boldsymbol{C M R} \leq 0.5$ and $1 / \eta_{1}$ is fixed. Thus, the probability of meeting the adaptive

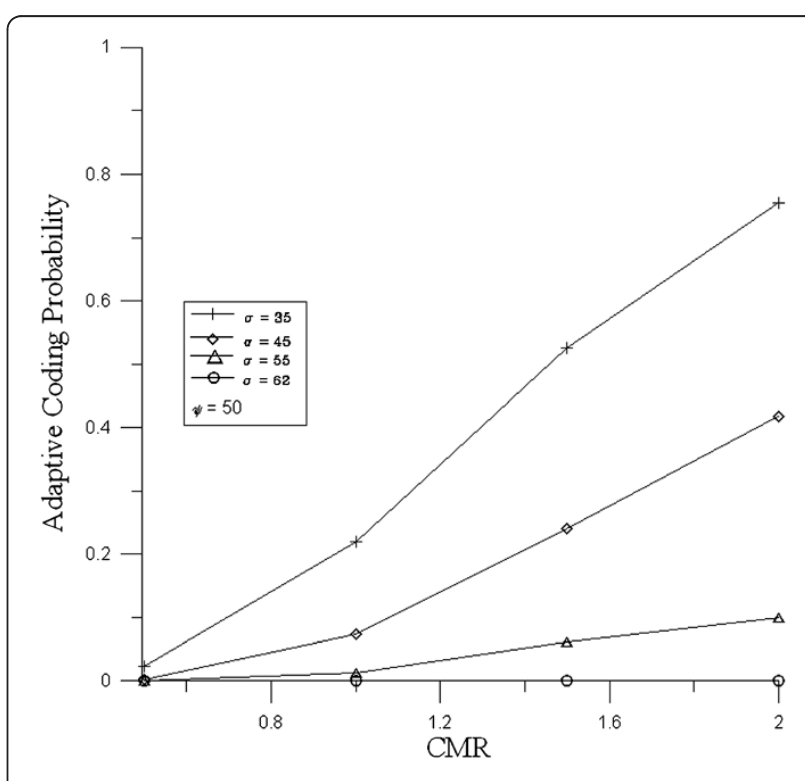

Figure 6 Adaptive coding probability versus the CMR of cell 1 . 
coding condition $\left(\sum_{s^{\prime} \in S} m_{i^{\prime}} b_{s^{\prime}}+b_{2}>\sigma\right)$ is very low. At this point, the effect of the adjustment of $\sigma$ on the adaptive coding probability is not significant. In other words, as the amount of new calls decreases, the adaptive coding probabilities of the four cases are close to zero when $\boldsymbol{C M R} \leq 0.5$. The dropping probabilities of handover connection requests for services will be increased when CMR increases, as shown in Figure 5. This indicates that the occupancy of cell 1 becomes higher and the probability of meeting the adaptive coding condition $\left(\sum_{s^{\prime} \in S} m_{i^{\prime}} b_{s^{\prime}}+b_{2}>\sigma\right)$ is higher. At this point, the adaptive coding probabilities of handover connection requests for type 2 services are higher, as shown in Figure 6. However, Figure 6 also shows that the adaptive coding probability increases when CMR and $\psi$ are fixed, but $\sigma$ is decreased, which is the same relationship as that shown in Figure 3.

Figure 7 shows the average PSNR of handover connection requests for type 2 services after the CMR of types 1 and 2 services is increased. The adaptive coding probability of handover connection requests for type 2 services will increase when the CMR increases, as shown in Figure 6. The average PSNR of handover connection requests for type 2 services is decreased when the adaptive coding probability is increased, as shown in Figure 4. Therefore, the average PSNR is gradually decreased while the CMR is gradually increased. In contrast, the average PSNR declines if $\sigma$ is lower, as shown in Figure 4. Thus, we can obtain show that the average PSNR becomes significantly lower if $\sigma$ is lower and CMR is fixed.

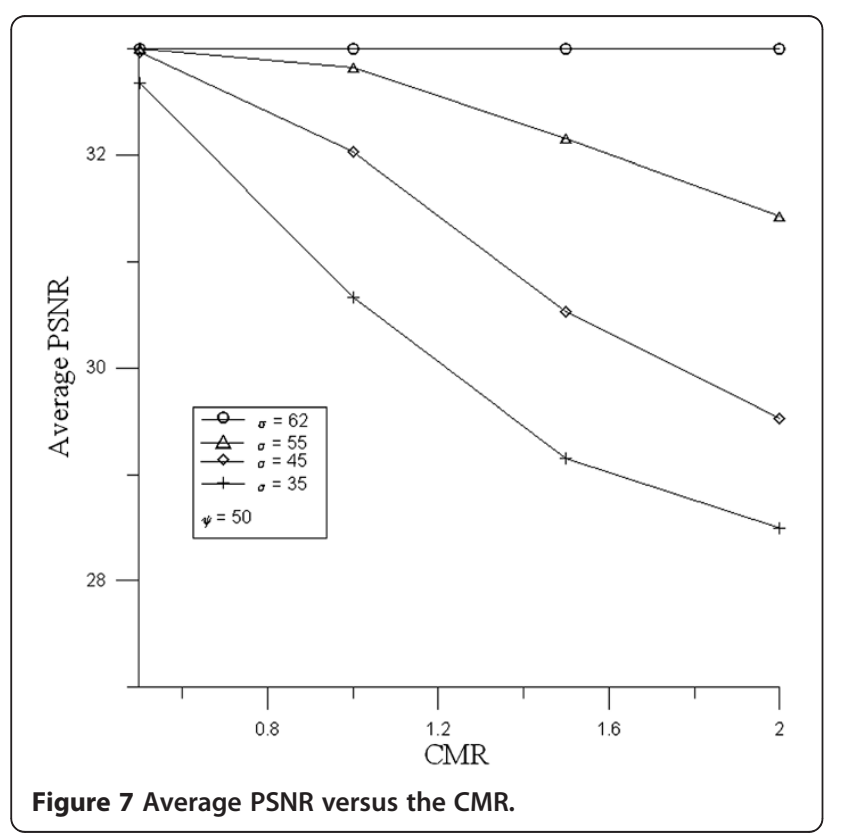

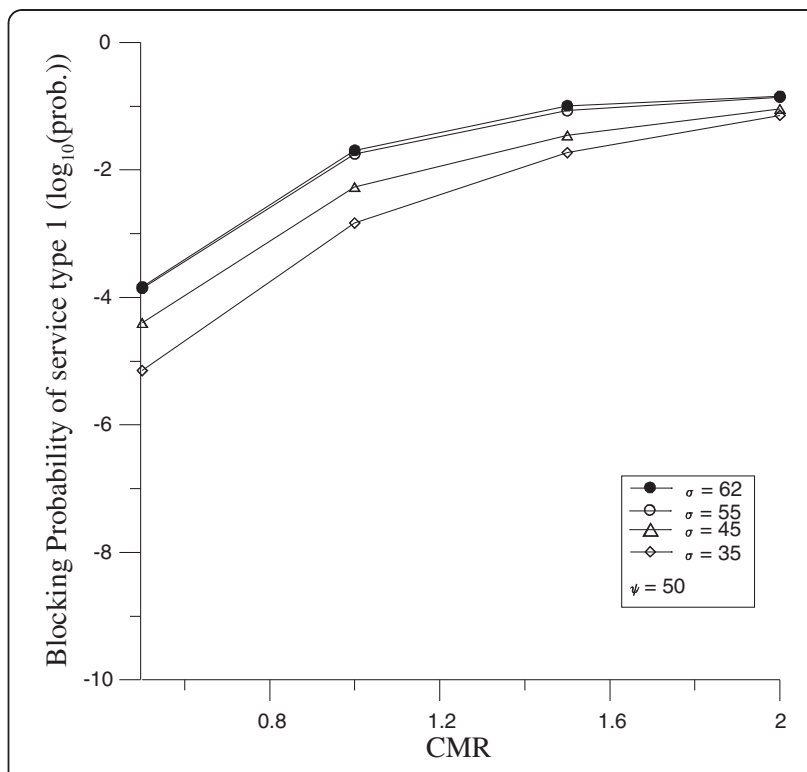

Figure 8 Effect of the CMR on the blocking probability of type 1 services.

Figures 8 and 9 show the blocking and forced termination probabilities of connection requests for type 1 services versus the CMR, respectively. The difference in the dropping probabilities of handover connection requests for type 1 services between $\sigma=62$ and 55 is very large, as shown in Figure 9. The occupancy of cell 1 decreases while type 2 service connections are changed into type 2* services at the point of handover. Next, the dropping probabilities of handover connection requests for types 1 and 2 services become lower, especially for type 1

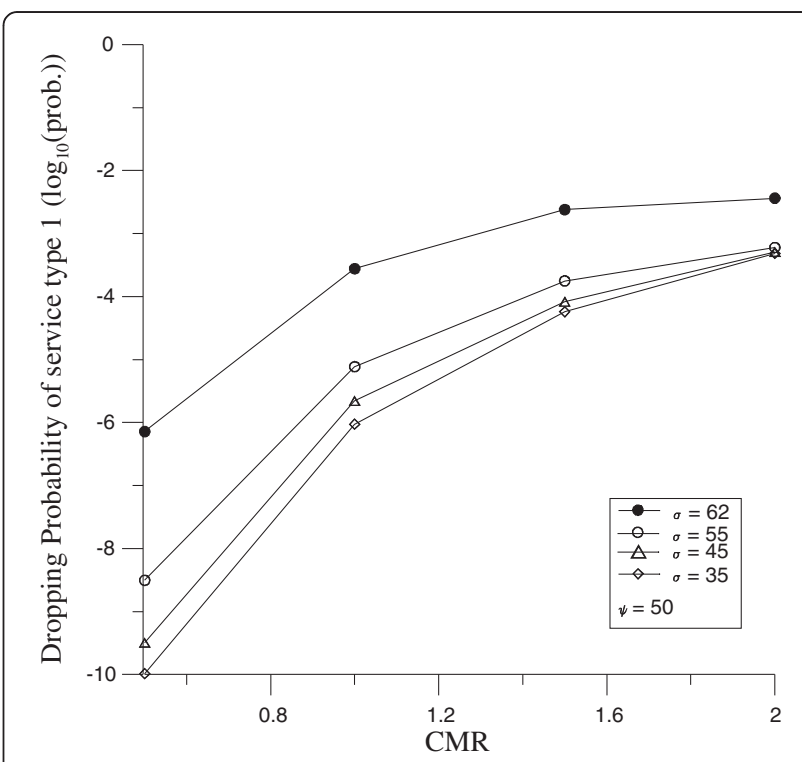

Figure 9 Effect of the CMR on the dropping probability of type 1 services. 


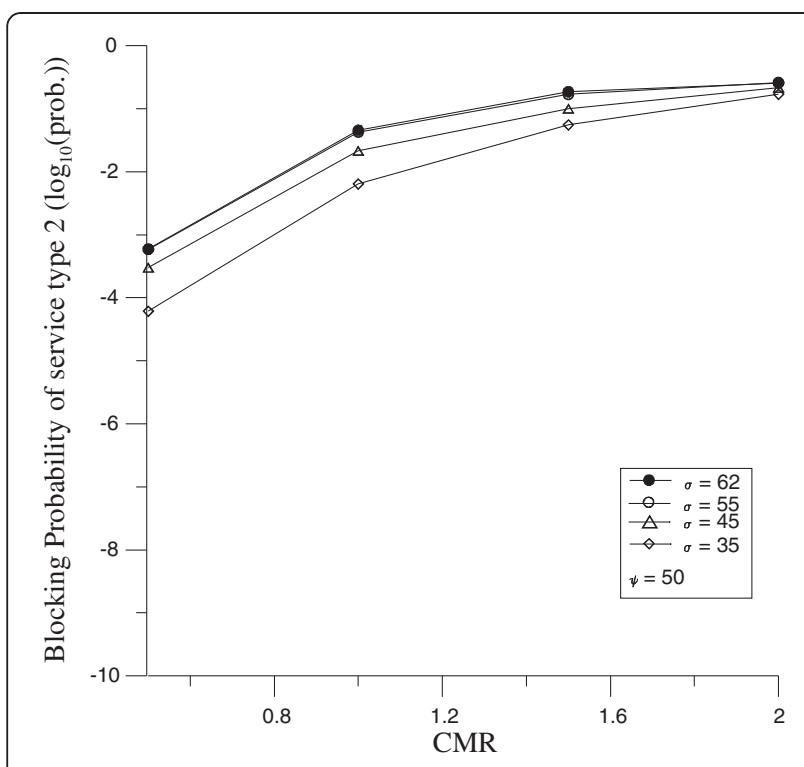

Figure 10 Effect of the CMR on the blocking probability of type 2 services.

services. This is because the bandwidth of type 1 service handover connection requests is lower than that of type 2 services.

Figures 10 and 11 show the blocking and forced termination probabilities of type 2 service connection requests versus the CMR, respectively. Figure 12 shows the forced termination probabilities of type $2^{*}$ service connection requests versus the CMR. $\sigma$ ranges from 35 to 62 , while $\psi$ is fixed at 50 . As shown in these figures, the CMR has a significant impact on the blocking and

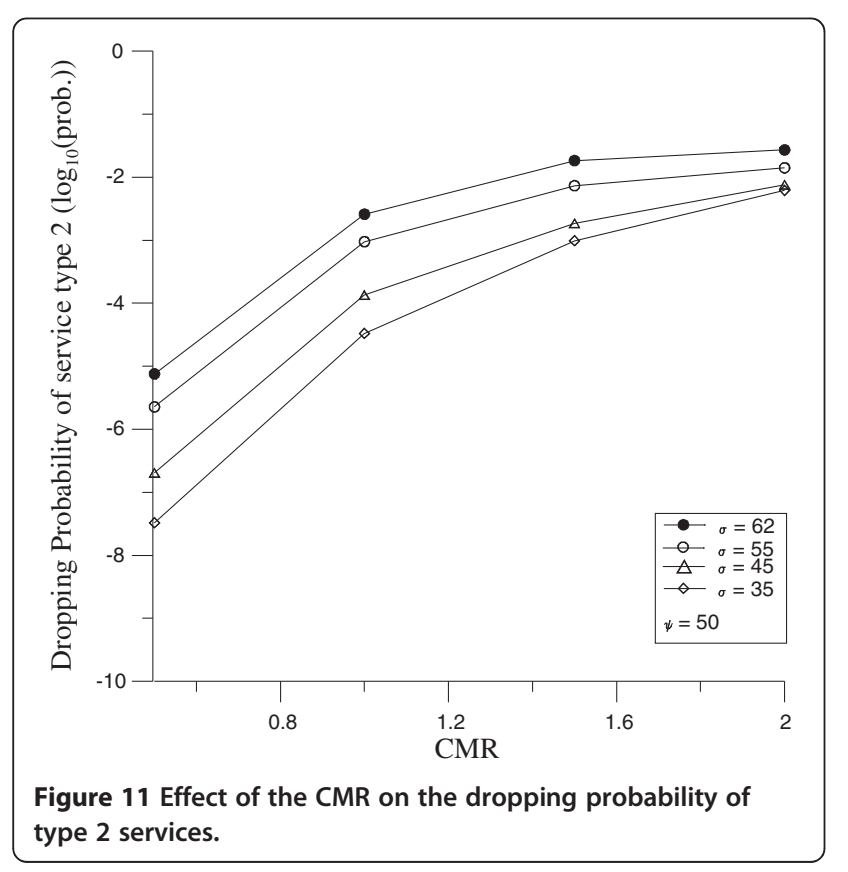

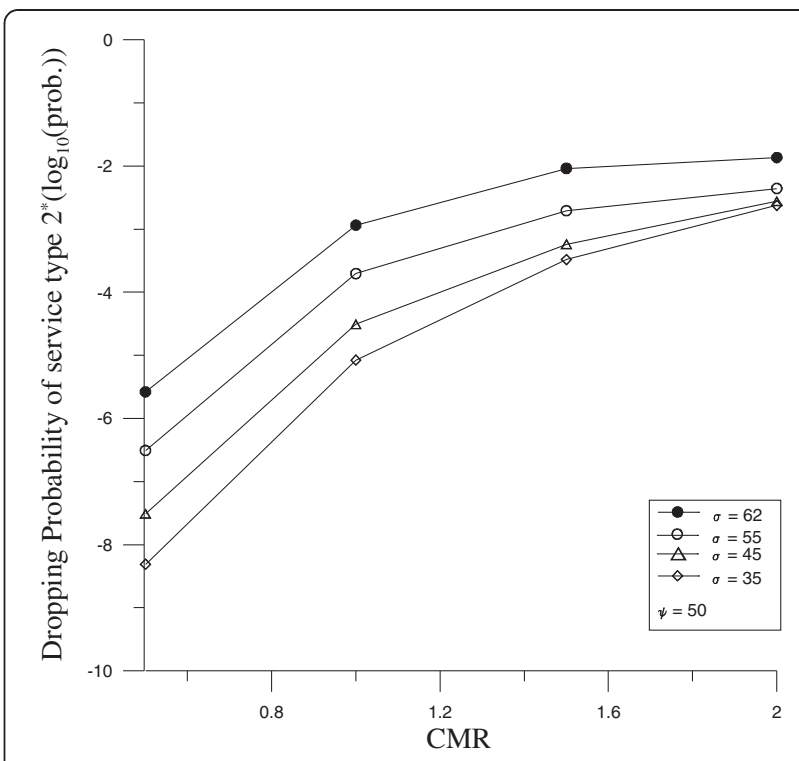

Figure 12 Effect of the CMR on the dropping probability of type $2^{*}$ services.

forced termination probabilities. As the CMR increases, there is a rapid increase in both probabilities. As the CMR approaches 2, the blocking probabilities of both services are close to 0.1 , and the forced termination probability is around $1 \times 10^{-3}$. The occupancy of cell 1 becomes lower and the blocking and forced termination probabilities are reduced due to the adaptive coding of type 2 services. Thus, user mobility can seriously affect the connection-level QoS, as shown in Figures 8, 9, 10, 11 , and 12.

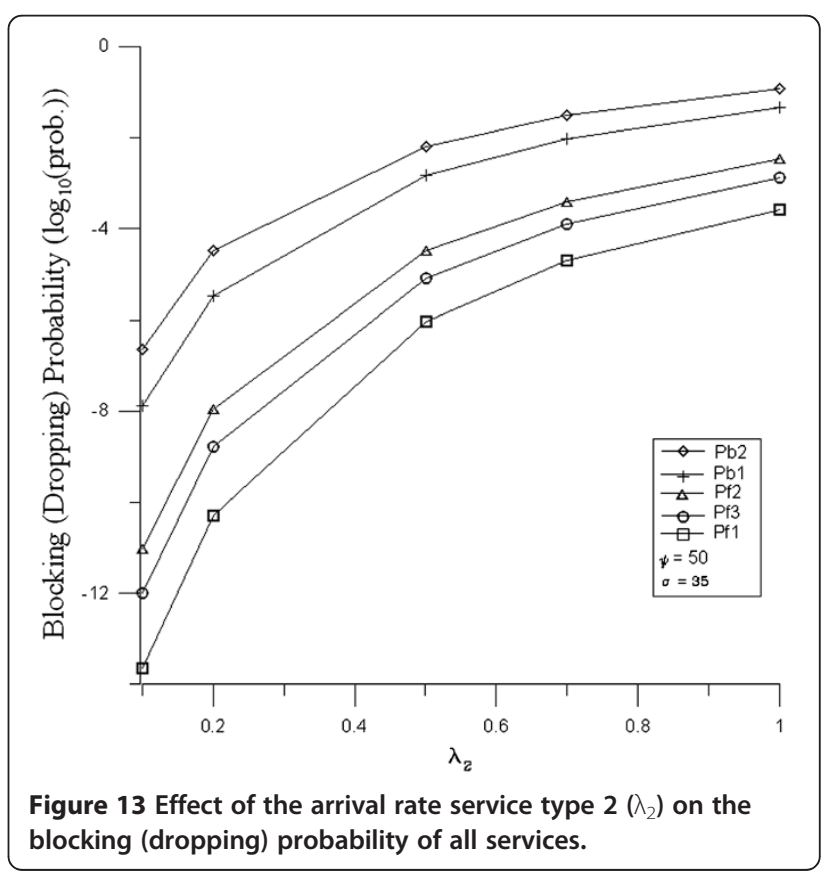


Varying arrival rate service type $2\left(\lambda_{2}\right)$

Figure 13 shows the connection-level QoS for the blocking and forced termination probabilities of both classes of calls versus the arrival rate of new calls for type 2 services $\left(\lambda_{2}\right)$. The new connection request threshold $(\psi)$ is 50 and the adaptive coding threshold $(\sigma)$ is 35 . The parameters $1 / v_{1}=1 / \nu_{2}=8 \mathrm{~min}$ and $1 / \eta_{i}=2 \mathrm{~min}$ are used. The arrival rate of new connection requests from type 1 services $\left(\lambda_{1}\right)$ remains constant at 0.5 connection requests per minute. $\lambda_{2}$ ranges from 0.1 to 1 . The blocking and forced termination probabilities are very sensitive to the arrival rate when the arrival rate is below a certain threshold (light load region).

This indicates the effectiveness of $\psi$ and $\sigma$ in providing a better connection-level QoS for more important handover calls.

Figures 14 and 15 show the blocking and forced termination probabilities of type 2 service connection requests versus $\lambda_{2}$, respectively. As $\sigma$ decreases, we can see that both probabilities decrease. More type 2 service connection requests of are easily changed to type $2^{*}$ service connection requests when $\sigma$ is lower. The goal of cell capacity saving can be met and the blocking and forced termination probabilities are reduced.

\section{Conclusion}

We developed a method for reducing blocking and dropping probabilities. The method used adaptive coding of handover connection service requests and the parameter adjustment of CAC policy functions in a multi-service cellular system. Mobility and handovers are

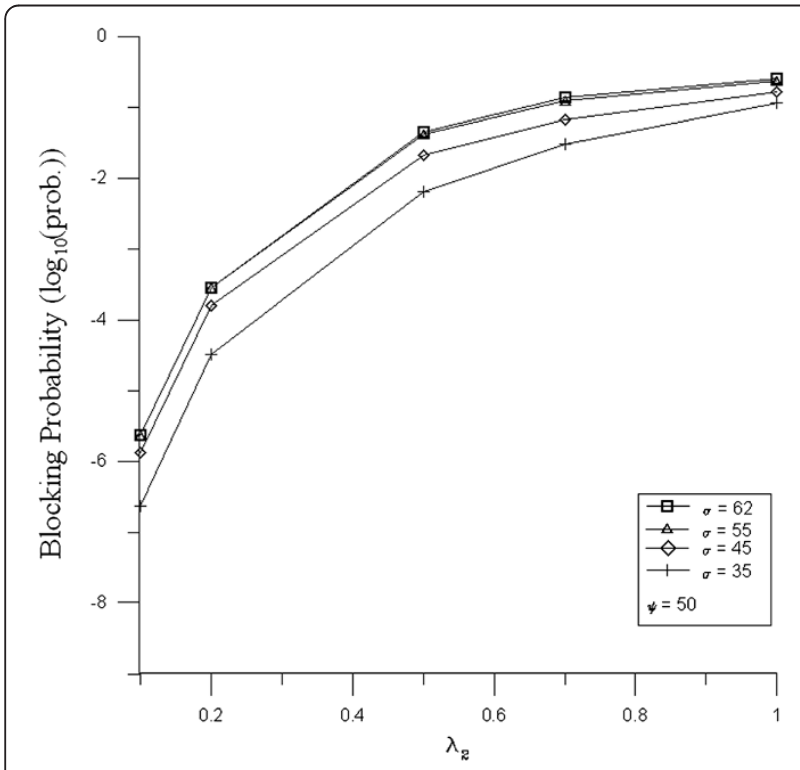

Figure 14 Effect of the type 2 service arrival rate $\left(\lambda_{2}\right)$ on the blocking probability of type 2 services.

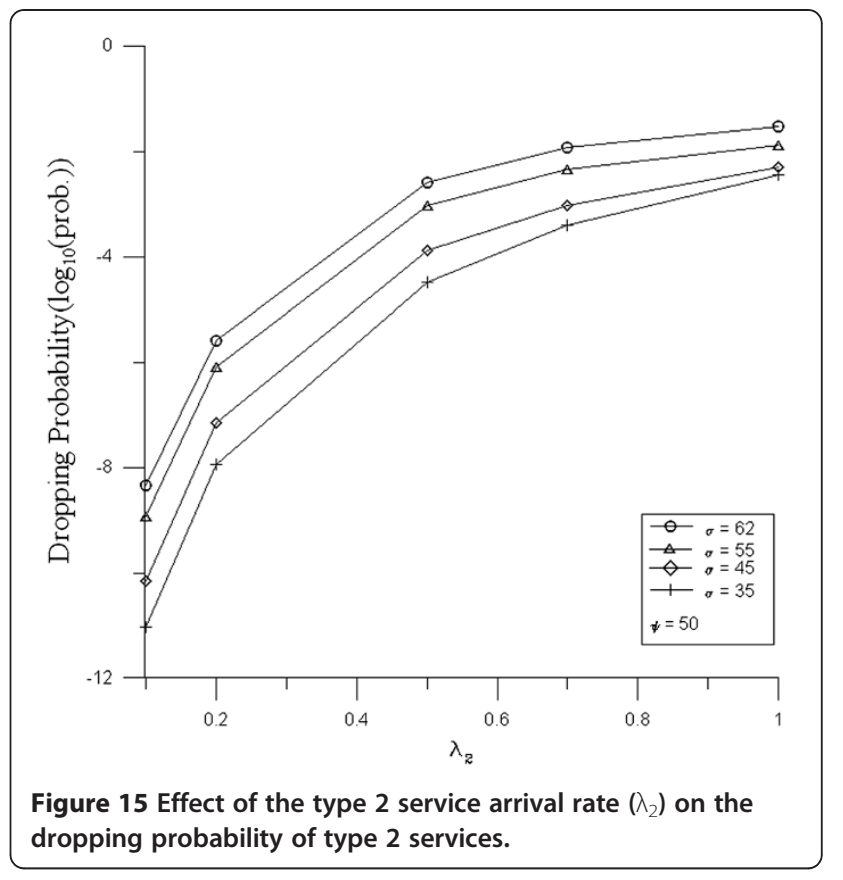

important factors in cellular systems, so multiservices based on models with user mobility and a CAC policy were considered. Our study considered the mobility and arrival (departure) rate of user connection requests, the capacity of each cell, CAC policy, the adaptive coding probability of the "dual mode full-rate service type", and blocking (dropping) probabilities of connection requests for each service. An efficient method is proposed to derive a better connection-level QoS, which was verified by performance simulations. The results indicate that the reservation policy for the new connection requests threshold $\psi$ and the adaptive coding threshold $\sigma$ were effective in providing a better connection-level QoS for new and handover calls. As an example in our study, we can see that in cell 1 , the new call dropping probability for type 1 services falls from $10^{-1.691}$ to $10^{-3.366}$ while for type 2 services fall from $10^{-1.342}$ to $10^{-2.702}$ due to $\sigma$. Furthermore, the handover call dropping probability for type 1 services drops from $10^{-3.557}$ to $10^{-6.366}$, type 2 services drops from $10^{-2.589}$ to $10^{-5.054}$, and type $2^{*}$ services fall from $10^{-2.939}$ to $10^{-5.584}$ due to $\sigma$. Therefore, the proposed method can satisfy the requisite connection-level QoS in multi-service cellular systems. Future work will study a CAC for AMR services with integrated macro/ femto cells. Various admission policies and their combinations will also be considered.

\section{Competing interests}

The authors declare that they have no competing interests.

\section{Acknowledgments}

The authors would like to thank the anonymous reviewers for their valuable comments and suggestions, which have greatly helped to improve the 
quality of this work. In addition, the authors thank the National Science Council of the Republic of China, Taiwan for financially supporting this research under Contract No. NSC100-2221-E-194-050

Received: 23 December 2011 Accepted: 18 May 2012 Published: 21 June 2012

\section{References}

1. L Huang, S Kumar, C-C Jay Kuo, Adaptive resource allocation for multimedia services in wireless communication networks, in International Conference on Distributed Computing Systems Workshop (ICDCSW '01), Mesa, AZ, USA, April 2001, pp. 307-312

2. D.G. Stratogiannis, G.I. Tsiropoulos, J.D. Kanellopoulos, P.G. Cottis, Probabilistic call admission control in wireless multiservice networks. IEEE Commun. Lett. 13(10), 746-748 (2009)

3. Y. Fang, Y. Zhang, Call admission control schemes and performance analysis in wireless mobile networks. IEEE Trans. Veh. Technol. 51(2), 371-382 (2002)

4. S. Tang, W. Li, Performance analysis of the $3 \mathrm{G}$ network with complementary WLANs, in IEEE Global Telecommunications Conference (GLOBECOM '05), St. Louis, MO 5, 2637-2641 (2005)

5. E. Stevens-Navarro, V.W.S. Wong, Resource sharing in an integrated wireless cellularMLAN system, in Canadian Conference on Electrical and Computer Engineering (CCECE 2007) Nancouver, BC, Canada, 2007), pp. 631-634

6. C.-C. Chao, W. Chen, Connection admission control for mobile multiple-class personal communications networks. IEEE J. Sel. Areas Commun. 15(8), 1618-1626 (1997)

7. E. Stevens-Navarro, A.-H. Mohsenian-Rad, V.W.S. Wong, Connection admission control for multi-service integrated cellular/WLAN system. IEEE Trans. Veh. Technol. 57(6), 3789-3800 (2008)

8. K. Homayounfar, Rate adaptive speech coding for universal multimedia access. IEEE Signal Process. Mag. 20, 30-39 (2003)

9. H. Holma, A. Toskala (eds.), WCDMA for UMTS (Wiley, New York, 2001

10. C.-H. Ke, C.-K. Shieh, W.-S. Hwang, A. Ziviani, Improving video transmission on the Internet. IEEE Potentials 26, 16-19 (2007)

11. C.M. Barnhart, J.E. Wieselthier, A. Ephremides, Admission control policies for multihop wireless networks. Wirel. Netw. 1(4), 373-387 (1995)

12. M. Naghshineh, M. Schwartz, Distributed call admission control in mobile/ wireless networks. IEEE J. Sel. Areas Commun. 14(4), 711-717 (1996)

13. I.F. Akyildiz, W. Wang, A dynamic location management scheme for nextgeneration multitier PCS systems. IEEE Trans. Wirel. Commun. 1(1), 178-189 (2002)

14. G. Bolch, S. Greiner, H. deMeer, K. Trivedi, Queueing Networks and Markov Chains: Modeling and Performance Evaluation with Computer Science Applications (Wiley, Hoboken, NJ, 1998)

15. K. Ross, Multiservice Loss Models for Broadband Telecommunication Networks (Springer-Verlag, New York, 1995)

16. 3GPP, Circuit Bearer Services Supported by a Public Land Mobile Network, TS $22.002(v 7.0 .0)(2007)$

17. D.Z. Deniz, N.O. Mohamed, Performance of CAC strategies for multimedia traffic in wireless networks. IEEE J. Sel. Areas Commun. 21(10), 1557-1565 (2003)

18. F.H.P. Fitzek, M. Reisslein, Video traces for network performance evaluation (2003). http://trace.eas.asu.edu/MDC/OCIF/foreman.html, Fall

19. 3GPP, Codecs for circuit switched multimedia telephony service, TS 26.110 (v7.0.0) (2006)

20. S. Morelli, A. Giordano, D. Giansanti, Routine tests for both planning and evaluating image quality in tele-echocardiography. Ann Ist Super Sanita 45(4), 378-391 (2009)

21. F.H.P. Fitzek, B. Can, R. Prasad, M. Katz, Traffic analysis and video quality evaluation of multiple description coded video services for fourth generation wireless IP networks. Wirel. Personal Commun. 35(1-2), 187-200 (2005)

22. I.F. Akyildiz, J.S.M. Ho, Y.-B. Lin, Movement-based location update and selective paging for PCS networks. IEEE/ACM Trans. Netw. 4(4), 629-638 (1996)

23. R. Jain, Y.-B. Lin, C. Lo, S. Mohan, A caching strategy to reduce network impacts of PCS. IEEE J. Sel. Areas Commun. 12(8), 1434-1444 (1994)

24. Y.-B. Lin, Mobility management for cellular telephony networks. IEEE Parallel Distrib. Technol. 4, 65-73 (1996) doi:10.1186/1687-1499-2012-199

Cite this article as: Wang and Pan: Admission control policy for adaptive multirate multiservices in cellular systems. EURASIP Journal on Wireless Communications and Networking 2012 2012:199.

\section{Submit your manuscript to a SpringerOpen ${ }^{\odot}$ journal and benefit from:}

- Convenient online submission

$\checkmark$ Rigorous peer review

- Immediate publication on acceptance

- Open access: articles freely available online

- High visibility within the field

- Retaining the copyright to your article

Submit your next manuscript at $>$ springeropen.com 